\title{
Das Projekt «Röntgenqualität in der Arztpraxis»
}

Jens-Uwe Schaaf

Dr. med., Facharzt für Radiologie, FMH, Ärztlicher Leiter der swissradiology consulting

In den Jahren 2014-2015 wurden in der Deutschschweiz insgesamt ca. 8000 Röntgenaufnahmen aus Praxen von Grundversorgern auf Einhaltung der standardisierten Qualitätskriterien von Einstelltechnik und radiologischer Beurteilbarkeit hin bewertet. Einige Praxen haben die definierten Qualitätskriterien sofort erfüllt, andere konnten zu der erforderlichen Qualitätsverbesserung ihrer Röntgenuntersuchungen geführt werden. Eine schweizweite Ausweitung des Projektes ist geplant.

\section{Der allgegenwärtige Qualitätsbegriff}

Die Ökonomisierung des Gesundheitswesens und ein steigendes Kostenbewusstsein haben in den letzten Jahren zu einem zunehmenden Gebrauch von Qualitätsbegriffen in der Medizin geführt. Nur noch selten findet man Geschäftsberichte von Gesundheitsorganisationen, in denen diese keine zentrale Rolle spielen.
Lesen Sie hierzu auch das anschliessende Interview mit Jens-Uwe Schaaf.
Immer mehr Krankenhäuser und Praxen lassen, wie man es seit Jahren bereits aus der Industrie kennt, das Management, die Organisation und die Arbeitsabläufe durch externe Stellen prüfen und zertifizieren. Der Erwerb eines Zertifikates dient zum einen der Verbesserung des Qualitätsbewusstseins bei den Mitarbeitern der Unternehmen und der Verbesserung der Arbeitsabläufe, zum anderen ist es eine Visitenkarte für die Patienten und Versicherungen.

Die meisten Zertifikate beziehen sich jedoch auf das Managementsystem und die Organisation der Gesundheitsunternehmen. Zertifizierte Prüfungen, die die Qualität der medizinischen Leistung bewerten, gibt es wenige. Ein Grund hierfür ist, dass es vielfach unmöglich ist, die Qualität eines Behandlungsergebnisses objektiv zu bewerten. Auch kommt es auf den Standpunkt des Betrachters an, der den Qualitätsbegriff definiert. Ist es der Patient, der eine maximale Dienstleistung erwartet, der Versicherer, der auf Outcome und Kosten schaut, oder der Arzt, der aufgrund seiner Qualifikation, die auf seiner Ausbildung und der persönlichen Erfahrung beruht, das Machbare als Einziger wirklich beurteilen kann? Der Begriff «Qualität» sollte bei der Beurteilung von medizinischen Leistungen nur von fachlich kompetenten Personen verwendet werden und nur dann, wenn die bewerteten Leistungen anhand von Standards messbar sind.

\section{Gut messbare Ergebnisqualität}

Radiologische Untersuchungen, zu denen auch das konventionelle Röntgen gehört, zählen zu den medizi- 
nischen Leistungen, bei denen die Ergebnisqualität gut messbar ist. Die Qualität einer Röntgenuntersuchung ist durch Standards definiert.

Einfluss auf die Qualität einer Röntgenuntersuchung haben die technischen Voraussetzungen, bedingt durch Geräte- und Einstelltechnik, sowie die Befundqualität, die über Indikation, Anamnese, Fragestellung und Erfahrung des Arztes in der Beurteilung konventioneller Röntgenbilder definiert wird.

Die Diagnose, die sich aus der Beurteilung eines konventionellen Röntgenbildes ergibt, ist, neben der klinischen Untersuchung, häufig eine wichtige Grundlage für therapeutische Entscheidungen im Praxisalltag. Ist die Qualität einer Röntgenuntersuchung eingeschränkt, besteht die Gefahr einer falsch positiven oder falsch negativen Diagnose. Eventuell werden auch zusätzliche Untersuchungen angefertigt, die mit einer weiteren Strahlenbelastung des Patienten verbunden sind, die bei Einhaltung der definierten Qualitätskriterien für die Röntgenuntersuchung nicht notwendig gewesen wären.

Insgesamt wurden in der Schweiz im Jahre 2008 über 6 Millionen konventionelle Röntgenbilder angefertigt, Tendenz steigend. War die durch konventionelle Röntgenaufnahmen verursachte Strahlenbelastung pro Einwohner in der Schweiz im Jahre 2008 noch 0,36 mSv, so betrug sie im Jahre 2013 bereits $0,41 \mathrm{mSv}$

Etwa 38,8\% aller radiologischen Untersuchungen sind konventionelle Röntgenaufnahmen von Thorax, Abdomen und peripherem Skelett. Sie zählen damit, neben dem Röntgen der Zähne, zu den am häufigsten durchgeführten radiologischen Untersuchungen. Trotz dieses hohen Anteils beträgt die durch konventionelle Röntgenaufnahmen verursachte Strahlenbelastung nur 10,7\% der Gesamtdosis durch radiologische Untersuchungen und liegt damit weit unter dem Anteil von 70,5\% der CT-Untersuchungen.

In der Schweiz betreiben ca. 5500 Ärzte, die keine Radiologen sind, eine Röntgenanlage. Davon sind etwa 4000 Grundversorger. Der grösste Teil der Röntgenaufnahmen wird in den Praxen und zunehmend auch in den Ambulanzen der Spitäler von Medizinischen Praxisassistentinnen (MPA) durchgeführt. Für die MPA ist die konventionelle Radiologie nur ein Teil ihrer sehr umfangreichen Ausbildung.

\section{Das Projekt «Röntgenqualität in der Arztpraxis»}

Trotz der Möglichkeit, die Qualität von Röntgenuntersuchungen zu messen, gibt es derzeit in der Schweiz keine institutionalisierte Qualitätskontrolle. Dass hier- für durchaus Bedarf besteht, zeigt nicht nur eine gewisse Anzahl von Röntgenaufnahmen, die bei externen Überweisungen von Patienten in den Kliniken wiederholt werden, sondern auch die zunehmend positive Resonanz durch die an dem Projekt «Röntgenqualität in der Arztpraxis» der swissradiology consulting teilnehmenden Ärzte und MPA und die positiven Ergebnisse, die mit diesem Projekt in den letzten zwei Jahren erzielt werden konnten.

Die Kernkompetenz der swissradiology consulting liegt im Qualitätsmanagement Radiologie. Dabei haben sich in den letzten Jahren zahlreiche Kooperationen mit Einzelpraxen, Gesundheitszentren und Ärztenetzwerken entwickelt. Neben Fortbildungsveranstaltungen für radiologisch tätige Berufsgruppen, der Erstellung von Zweitmeinungen zu radiologischen Untersuchungen durch das Experten-Team Radiologie, unterstützt die swissradiology consulting seit zwei Jahren mit dem Projekt «Röntgenqualität in der Arztpraxis» radiologisch tätige Grundversorger bei der Qualitätssicherung in der konventionellen Röntgendiagnostik.

In der Schweiz betreiben ca. 5500 Ärzte, die keine Radiologen sind, eine Röntgenanlage.

Im Jahr 2014 erfolgte auf Initiative des ärztlichen Direktors einer Gruppe von Gesundheitszentren die Implementierung des Projektes «Röntgenqualität in der Arztpraxis» in 20 Gemeinschaftspraxen der Ostschweiz, um die Röntgenqualität in den Praxen auf ein mögliches Verbesserungspotential hin zu überprüfen. In den Jahren 2014/2015 wurden in den Gemeinschaftspraxen in zwei Teilabschnitten des Projektes über 5000 Röntgenaufnahmen jeweils auf korrekte Einstelltechnik und radiologische Beurteilbarkeit durch ein Team, bestehend aus einem erfahrenen MTRA und Radiologen, im Vieraugenprinzip bewertet. Die Bewertungskriterien sind definiert und stützen sich auf internationale Standards, die in der Röntgen-Ausbildung des Fachpersonals in der Schweiz gelehrt werden. Bei der Beurteilung wurde bei jedem Röntgenbild die Einhaltung von acht wichtigen Einzelkriterien der Einstelltechnik geprüft. Mit Bezug auf die radiologische Beurteilbarkeit erfolgte weiterhin die Zuordnung jedes Röntgenbildes zu einer von vier Qualitätsgruppen, von sehr gut bis insuffizient. Die häufigsten Mängel waren im Bereich der korrekten Einstelltechnik von Gelenken und im Bereich der Einblendung zu finden.

Die Zuordnung in den Bereich der insuffizienten, d.h. nicht korrekt beurteilbaren Röntgenaufnahmen erfolgte u.a. bei falscher Seitenbezeichnung bei Gelenken oder wenn der Untersuchungsbereich grob abgeschnitten war. Weiterhin wurden dieser Gruppe die 


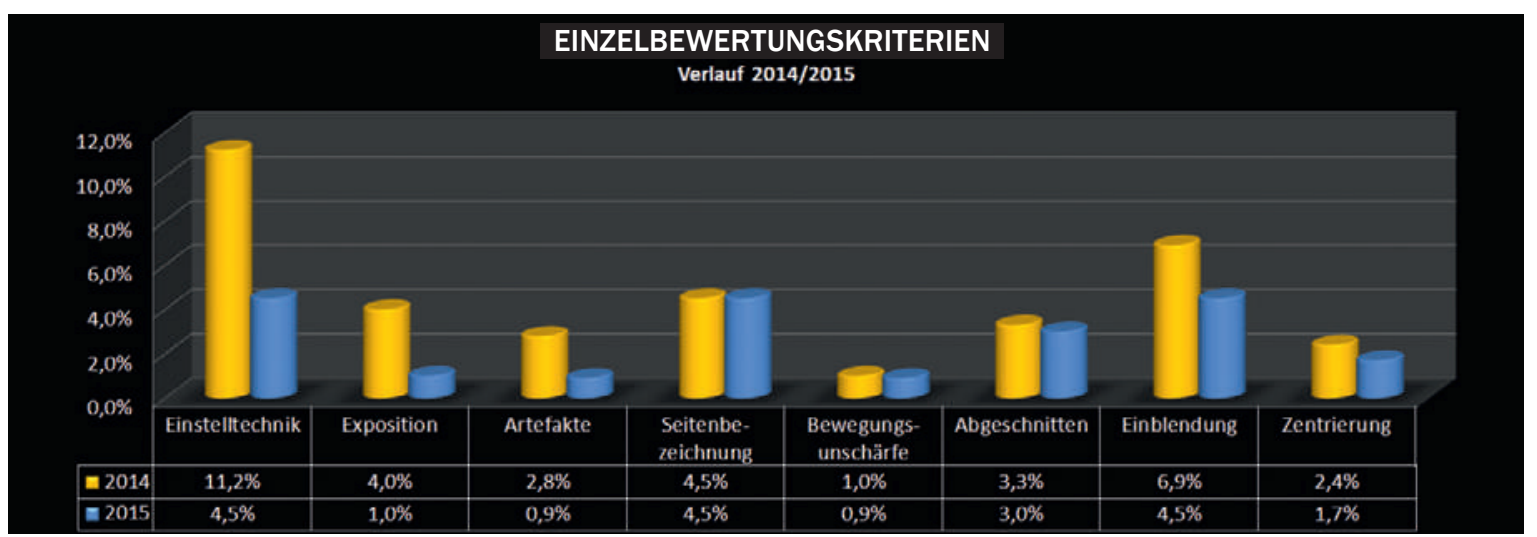

Einzelbewertungskriterien mit Ergebnissen in den Jahren 2014 und 2015.

Röntgenbilder zugeordnet, die aufgrund einer Überoder Unterbelichtung auch mit den Möglichkeiten der digitalen Bildnachverarbeitung nicht beurteilbar waren.

Für jede Praxis wurde ein übersichtlicher Qualitätsbericht mit beispielhaften Hinweisen für Verbesserungsmöglichkeiten bei der Einstelltechnik und bei technischen Mängeln, die die Bildqualität beeinflussten, erstellt. Der Umgang mit den Patientendaten und den Auswertungen ist durch eine Datenschutzerklärung der swissradiology consulting geregelt, die das Schweizer Recht beachtet.

Nach der ersten Teilauswertung des Projektes «Röntgenqualität in der Arztpraxis» hatten die Gemeinschaftspraxen über einen Zeitraum von 6-12 Monaten Gelegenheit, durch interne und externe Schulungen sowie vereinzelt notwendige technische Anpassungen der Röntgenanlagen, die Voraussetzungen für die zweite Teilauswertung zu schaffen.

\section{Ergebnisse}

Die Bewertung der Röntgenaufnahmen in den 20 am Pilotprojekt teilnehmenden Praxen im Jahr 2015 ergab im Vergleich zum Jahr 2014 in fast allen Praxen eine signifikante Qualitätsverbesserung. Sowohl die Anzahl der Aufnahmen mit mässiger Qualität wie auch die als insuffizient eingestuften waren dank der erfolgten Massnahmen deutlich gesunken.

Das Ergebnis des Pilotprojektes «Röntgenqualität in der Arztpraxis» mit der Auswertung von insgesamt 5000 Röntgenaufnahmen zeigt, dass der interdisziplinäre Ansatz zur Qualitätssicherung der Röntgenqualität erfolgreich sein kann. Mit ihrer Teilnahme an dem Projekt RQA leisten die Praxen und Gesundheitszentren einen aktiven Beitrag zur Qualitätssicherung in der Röntgendiagnostik. Das Projekt baut auf Kontinuität mit sich in zweijährigen Abständen wiederholenden Bewertungen der Röntgenqualität in der Arztpraxis. Die Finanzierung des Projektes erfolgt über Beiträge der teilnehmenden Praxen und/oder Ärztenetzwerke.

Die Erfahrung der an dem Projekt teilnehmenden MPA und Ärzte war sehr positiv. Letztlich wurde der Erfolg des Projektes nicht nur durch dessen Ergebnisse, sondern auch durch die Akzeptanz dokumentiert, die sich aus der Auswertung der Evaluationsbögen ergab, die nach Projektabschluss an die projektverantwortlichen MPA und Ärzte verschickt wurden.

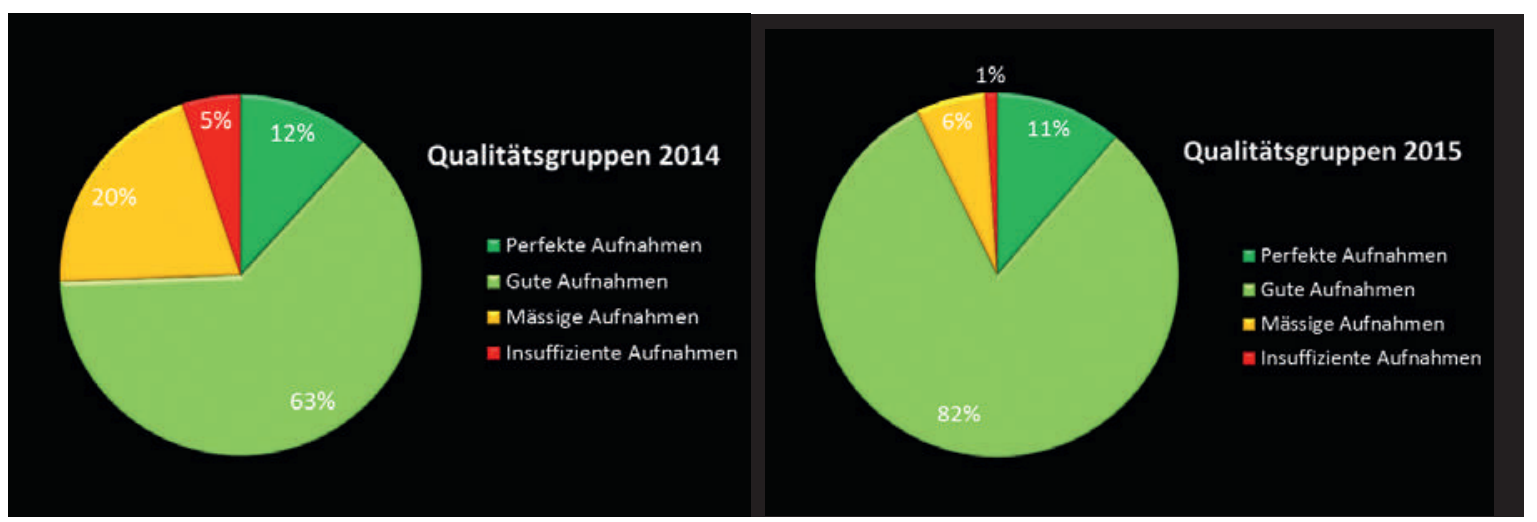

Gesamtbewertung in Qualitätsgruppen 2014-2015 von je 2500 Röntgenaufnahmen. 


\section{Rückmeldungen}

Folgende Bewertungen zu dem Projekt «Röntgenqualität in der Arztpraxis» wurden von MPAs vermerkt:

- Positiv, um zu wissen, wo wir stehen (santémed)

- In unserem Betrieb waren alle offen für diese Aufgabe und waren auch neugierig auf das Resultat um zu sehen, ob wir gut und qualitativ arbeiten und das sollte ja das eigentliche Ziel sein (Monvia)

- Positiv weitere Auswertung nach gewisser Zeit, gute Idee, danke (santémed)

- Gute Anregungen zur Verbesserung der Röntgenqualität (mediX)

- Mir hat das Projekt sehr gefallen und ich denke, eine regelmässige Qualitätskontrolle ist sinnvoll. Sehr gut fand ich auch, dass man einen Vergleich hat zu den anderen Zentren, um zu sehen, wo man steht und was verbessert werden kann. RX-Bilder werden ja auch an Spitäler weitergereicht und sind die "Visitenkarte» der MPA oder? Wurde berücksichtigt, dass auch Auszubildende röntgen und evtl. die Erfahrung noch etwas fehlt? (Monvia)

Das sagten Ärzte zu dem Projekt «Röntgenqualität in der Arztpraxis»:

- Ich finde diese Art von Qualitätsüberprüfung/Verbesserung sehr sinnvoll und hilfreich (santémed)

- Ausgezeichnetes Projekt für die Qualitätssicherung, relativ einfach machbar (mediX Bern)

- Qualitätsentwicklung hat in unserem Ärztenetzwerk einen hohen Stellenwert. Entsprechend danken wir für ihre wertvolle Arbeit. (Igomed)

- Ein Projekt, welches unserem Bestreben nach Qualitätssicherung und gezieltem Mitarbeiter-Training sehr entgegenkommt (Kinderarzthaus St.Gallen)

Anfang 2015 wurde das Projekt RQA der Schweizerischen Akademie für Qualität in der Medizin (SAQM) der FMH mit der Bitte um inhaltliche Unterstützung vorgestellt. Ziel war es, das Projekt von seinem Pilotstatus in eine breitere, schweizweite Anwendung zu überführen. Der Steuerungsausschuss der SAQM hat anlässlich seiner Sitzung vom 19. Februar 2015 das Projekt und dessen Inhalte als wertvoll für die Qualität der ärztlichen Leistungserbringung eingestuft und die Aufgleisung des Projektes als fundiert und professionell eingeschätzt.

Im September 2015 wurde das Managementsystem der swissradiology consulting, das auch das Projekt «Röntgenqualität in der Arztpraxis» beinhaltet, nach ISO 9001:2008 zertifiziert.

Die Anzahl der an dem Projekt «Röntgenqualität in der Arztpraxis» teilnehmenden Praxen hat sich in den Jah- ren 2014 und 2015 von ursprünglich 22 auf 50 erhöht. Einige der teilnehmenden Praxen haben bei der Bewertung der Röntgenqualität bereits beim ersten Mal die definierten Standards voll erfüllt. Zu den teilnehmenden Praxen und Gesundheitszentren, die 2015 das Zertifikat «Röntgenqualität» der swissradiology consulting erhalten haben, gehören u.a. Gesundheitszentren von santémed, Gesundheitszentren der Monvia AG, Gruppenpraxen von mediX Bern und das Kinderarzthaus St.Gallen. Die Privatklinikgruppe Hirslanden startete ihre Teilnahme im Oktober 2015 in einem Pilotprojekt mit einem ihrer Praxiszentren. Das Ärztenetzwerk Igomed unterstützt die Teilnahme seiner Mitglieder an diesem Projekt und übernimmt für die nächsten zwei Jahre 50\% der Kosten. Für das Jahr 2016 haben Ärztenetzwerke mit über 300 radiologisch tätigen Praxen ihre Teilnahme an dem Projekt «Röntgenqualität in der Arztpraxis» zugesagt.

Das Projekt beruht auf Freiwilligkeit, auf dem Prinzip des «Peer Review», d.h. Gespräche und Kommunikation finden mit den Kollegen auf Augenhöhe statt. Alle an dem Prozess der Erstellung von Röntgenbildern Beteiligten werden in das Projekt eingebunden. Mit der Auswertung der Evaluationsbögen werden die Abläufe des Projektes kontinuierlich verbessert.

Die Ergebnisse der Auswertungen werden streng vertraulich behandelt und nur den ärztlichen Leitern der teilnehmenden Praxen in anonymisierter Form ausgehändigt.

Die swissradiology consulting wird sich als unabhängige Organisation mit seinem Experten-Team Radiologie in den nächsten zwei Jahren für eine schweizweite Einführung des Projektes «Röntgenqualität in der Arztpraxis» zur Qualitätssicherung in der Röntgendiagnostik einsetzen.

\section{Referenzen}

1 Bundesanzeiger: Bekanntmachung eines Beschlusses des Gemeinsamen Bundesausschusses über die Neufassung der Qualitätsbeurteilungs-Richtlinie Radiologie: Anpassung an aktuelle Leitlinien vom 17. Juni 2010

2 Aroua A, Samara ET, Bochud FO, Francis, Verdun FR, Vader JP. Exposure of the Swiss population by Medical X-rays: 2008. Review Lausanne; June 2011.

3 Le Coultre R, Bize J, Champendal M, Wittwer D, Trueb P, Verdun FR. Exposition de la population suisse aux rayonnements ionisants en imagerie médicale en 2013. Federal Office of Public Health. Bern, Switzerland.

\section{Disclosure statement}

Der Autor ist ärztlicher Leiter der swissradiology consulting, eines privaten Dienstleistungsunternehmens im Bereich der medizinischen Radiologie, das unter anderem das Projekt «Röntgenqualität in der Arztpraxis» initiiert hat. 